\title{
Bio-inspired and pheromone-based shop-floor control
}

\author{
B. SCHOLZ-REITER, C. DE BEER*, M. FREITAG and T. JAGALSKI \\ University of Bremen, Bremen, Germany
}

\begin{abstract}
Autonomous control means the decentralized coordination of intelligent logistic objects and the routing through a logistic system by the intelligent parts themselves. This paper shows the application of a pheromone-based autonomous control method to a matrix model of a shop floor and compares the performance to an earlier developed method in different dynamic demand situations. The discrete event simulations are analysed by comparing statistics on throughput time data resulting from the system's behaviour in dynamic order arrival situations.
\end{abstract}

Keywords: Logistics; Autonomous control; Modelling; Simulation

\section{Introduction}

In recent manufacturing industry, production planning and control (PPC) systems have to cope with rising complexity and dynamics (Tönshoff et al. 2002, Kim and Duffie 2004, Scholz-Reiter et al. 2004c). One approach to meet these demands is to develop decentralized systems with autonomous control methods to reduce the amount of complexity that has to be taken into account for decision making (Scholz-Reiter et al. 2004b). Autonomous control means the decentralized coordination of intelligent logistic objects and the routing through a logistic system by the intelligent parts themselves. Therefore it is necessary to develop local decision rules that allow for local autonomous decision making while the global objectives are reached through interaction and emergent behaviour (Ueda et al. 2001, Parunak 1997). In earlier work the authors have developed two different local decision rules that allow for autonomous control (ScholzReiter et al. 2005, Armbruster et al. 2006). The latter is inspired by the behaviour of social insects and will be called pheromone based rule. The application of these rules to a shop floor scenario and their ability to cope with rising dynamics will be analysed and compared in this paper.

\section{Autonomy in production logistics}

The concept of autonomous control requires on one hand logistic objects that are able to receive local information, process this information, and make a decision about their next action. On the other hand, the logistic structure has to provide distributed information about local states and different alternatives to enable decisions generally. These features will be made possible through the development of ubiquitous computing technologies (Fleisch and Tellkamp 2005).

The application of autonomous control in production logistics can be realized by recent information and communication technologies such as radio frequency identification (RFID), wireless communication networks, etc. These technologies facilitate intelligent and autonomous parts and products which are able to communicate with each other and with their resources such as machines and transport systems and to process the acquired information. This leads to a coalescence of material flow and information flow and enables every item or product to manage and control its manufacturing process autonomously (ScholzReiter et al. 2004c). The coordination of these intelligent objects requires advanced PPC concepts and strategies to realize autonomous control of logistic processes. To develop and analyse such autonomous control strategies dynamic models are required. In the following a shop floor scenario introduced by Scholz-Reiter et al. (2004a) is modified and used to model autonomous processes in a flexible production scenario.

\section{Shop-floor scenario}

To compare the different autonomous control methods a shop floor scenario is needed that allows for the application

*Corresponding author. Email: ber@biba.uni-bremen.de

International Journal of Computer Integrated Manufacturing

ISSN 0951-192X print/ISSN 1362-3052 online (c) 2008 Taylor \& Francis

http://www.tandf.co.uk/journals

DOI: $10.1080 / 09511920701607840$ 
of autonomous control methods and is general enough to be valid for different classes of shop-floor types. For these reasons a shop floor model in matrix format has been chosen, see figure 1. Subsequent productions steps are modelled vertically while parallel stations are able to perform resembling processing steps.

At the source the raw materials for each product enter the system. Each product class has a different production plan, i.e. a list of processing steps that have to be fulfilled on the related machine. In case of overload the part can decide autonomously to change the plan and to use a parallel machine instead. The final products leave the system via drains.

\section{Autonomous control methods}

\subsection{Queue length estimator}

The first method called queue length estimator compares the actual buffer states at all the parallel machines that are able to perform its next production steps. Therefore the buffer content is not counted in number of parts but the parts are rated in estimated processing time and the actual buffer levels are calculated as the sum of the estimated processing time on the respective machine. When a part has to render the decision about its next processing step it compares the current buffer levels i.e. the estimated waiting time until processing and chooses the buffer with the shortest waiting time. The processing times are not fixed but fluctuate within a frame of $5 \%$. Therefore the mean values that are used to calculate the estimated waiting time is updated every five days for the queue length estimator.

\subsection{Pheromone method}

The second method does not use information about estimated waiting time, i.e. information about future events but uses data from past events. This method is inspired by the behaviour of foraging ants that leave a pheromone trail on their way to the food. Following ants use the pheromone trail with the highest concentration of pheromone to find the shortest path to the food. In the shop-floor scenario this behaviour is imitated in a way that whenever a part leaves a machine, i.e. after a processing step is accomplished, the part leaves information about the duration of processing and waiting time at the respective machine. The following parts use the data from the past five parts to render the decision about the next production step. This resembles the evaporation of the pheromone in reality and guarantees the up-to-dateness of the used data. The parts compare the mean throughput times from parts of the same type and choose the machine with the lowest mean duration of waiting and processing.

\section{Simulation model}

A simulation period of 30 days is chosen. After a phase of two months for avoiding transient effects the third month is used to measure the throughput time of every single part that is finished. For balancing conditions the minimal processing time per manufacturing step is equally $2 \mathrm{~h}$.

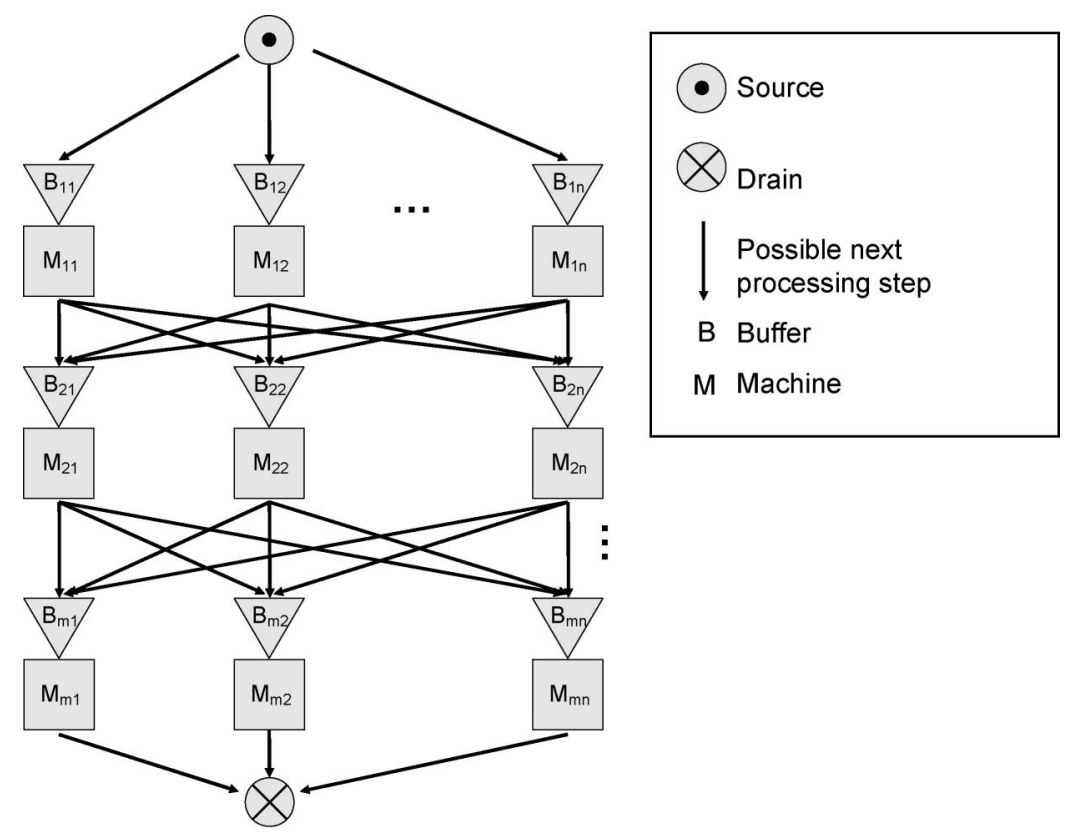

Figure 1. Matrix model of a shop floor. 
To model a highly dynamic market situation the demand for the different products is set as an oscillating curve with situation of over and under load. This means that an arrival rate of one part every $2 \mathrm{~h}$, i.e. an arrival rate of $0.5 \mathrm{l} / \mathrm{h}$ indicates a $100 \%$ utilization. For a mean utilization of $80 \%$ the arrival rate curves are set with a mean of $0.4 \mathrm{l} / \mathrm{h}$. The resulting arrival rate of parts per hour that enter the shop floor is shown in figure 2 .

Figure 3 shows the resulting time series for the throughput time of products for one product type if the queue length estimator is used for different amplitudes, i.e. varying dynamics in the arrival rate. One realizes that the rising amplitude has almost no effect on the through put time.

Figure 4 shows the corresponding time series for the same product type for the pheromone method. During periods of overload the throughput time rises higher than with the queue length estimator but the system learns and results in finding the shortest path for the parts and therefore in a low throughput time.

Figure 5 shows the resulting mean throughput time for all products for the two different methods and for different amplitudes. It appears that the pheromone method shows lower mean throughput times in situations of smaller amplitudes, i.e. less dynamics in the arrival rate. This is caused by the fact that the pheromone method needs some time to react on changing conditions and is less affective if the dynamics gets too high.

Secondly the better performance of the pheromone method in situations of lower dynamics is caused by the fact that the queue length estimator uses fuzzy information about future events i.e. estimated processing times. If the

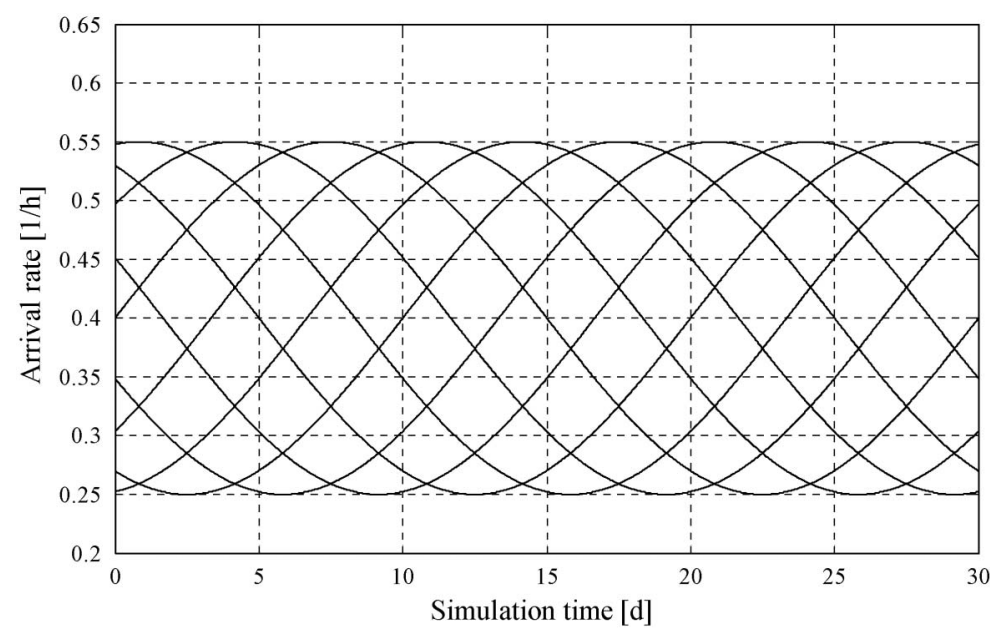

Figure 2. Arrival rate during one simulation period for nine different products.

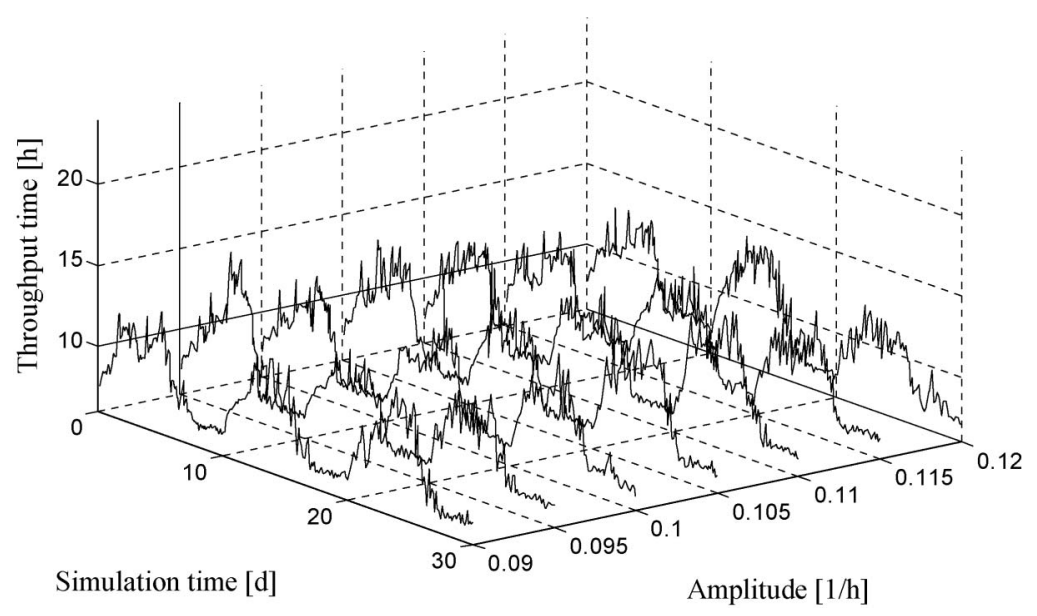

Figure 3. Throughput time for one product during one simulation period for different amplitudes using the queue length estimator. 


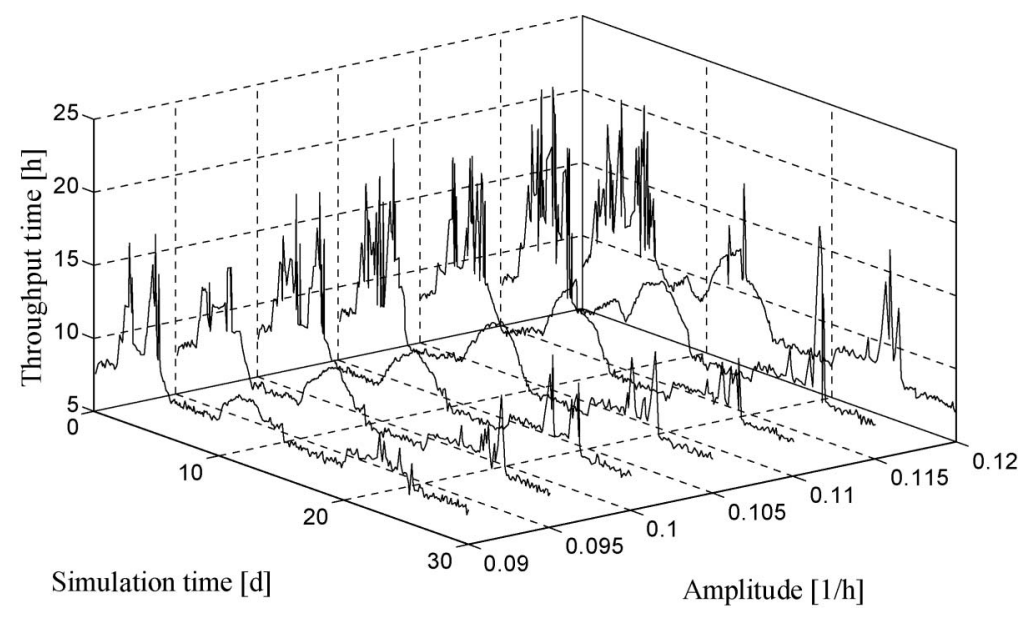

Figure 4. Throughput time for one product during one simulation period for different amplitudes using the pheromone rule.

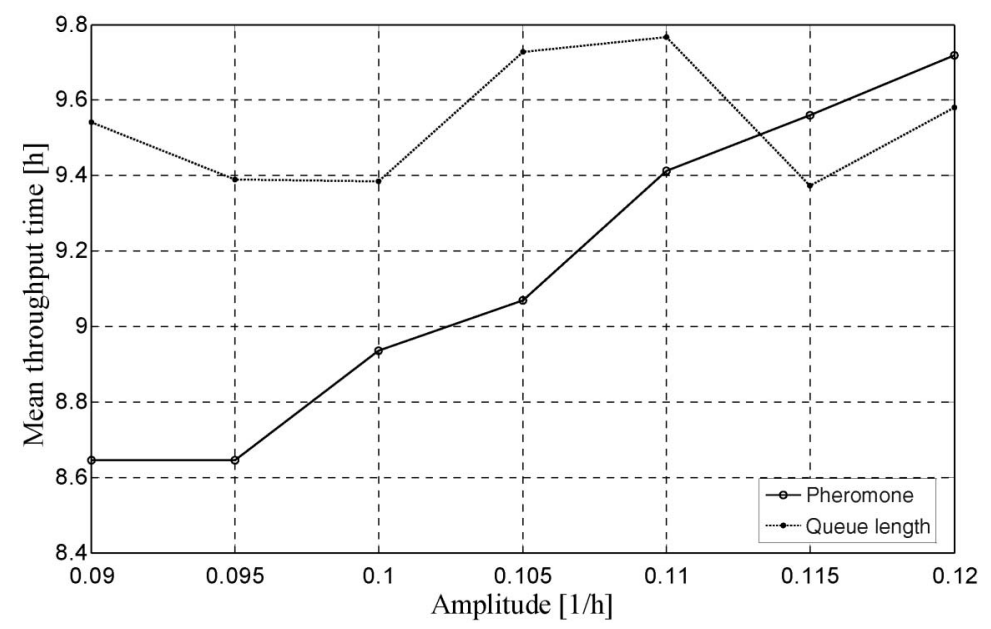

Figure 5. Comparison of the mean throughput times for the two different autonomous control methods.

processing times are exact and the queue length estimator has flawless data the performance of the queue length estimator is significantly better.

Therefore the result is that the pheromone method is advisable in situations of lower dynamics but high variance in processing times, here $5 \%$. Furthermore the advantage of pheromone based concepts often depends on a high number of elements that interact. The dependence of the results on the number of elements and the frequency of interaction will be the topic of future research.

\section{Acknowledgements}

This research is founded by the German Research Foundation (DFG) as part of the Collaborative Research Centre 637 'Autonomous Cooperating Logistic Processes:
A Paradigm Shift and its Limitations' (SFB 637) at the university of Bremen.

\section{References}

Armbruster, D., de Beer, C., Freitag, M., Jagalski, T. and Ringhofer, C., Autonomous control of production networks using a pheromone approach. Physica A, 2006, 363, 104-114.

Fleisch, E. and Tellkamp, C., The business value of ubiquitous computing technologies. In Ubiquitous Commerce, edited by G. Roussos, 2005 (Springer Verlag: New York).

Kim, J.-H. and Duffie, N.A., Backlog control for a closed loop PPC system. Annls CIRP, 2004, 53, 357-360.

Parunak, H., van Dyke, Go to the ant: engineering principles from natural multi agent systems. Annls Ops Res., 1997, 75, 69-101.

Scholz-Reiter, B., Freitag, M., de Beer, Ch. and Jagalski, Th., Modelling and analysis of autonomous shop floor control. Proceedings of the 38th CIRP International Seminar on Manufacturing Systems, Florianopolis, Brazil 2005. CD-ROM. 
Scholz-Reiter, B., Hamann, T., Höhns, H. and Middelberg, G., Decentral closed loop control of production systems by means of artificial neural networks. Proc. 37th CIRP-ISMS, Budapest, Hungary, 2004a, pp. 199 203.

Scholz-Reiter, B., Peters, K. and de Beer, C., Autonomous control of shop floor logistics. Chryssolouris. Manufacturing, Modelling, Management and Control 2004, IFAC Workshop Series, edited by D. Mourtzis, 2004b (Elsevier Science: Amsterdam).

Scholz-Reiter, B., Windt, K. and Freitag, M., Autonomous logistic processes: new demands and first approaches. Proc. 37th CIRP-ISMS, Budapest, Hungary, 2004c, pp. 357-362.
Tönshoff, H.K., Herzog, O., Timm, I.J. and Woelk, P.-O., Integrated process planning and production control based on the application of intelligent agents. Proc. 3rd CIRP ICME, 2002, pp. 135-140.

Ueda, K., Markus, A., Monostori, L., Kals, H.J.J. and Arai, T., Emergent synthesis methodologies for manufacturing. CIRP Annls., 2001, 50, 535-551. 